\title{
ABSTRACT \\ THE SIMULTANEOUS USE OF MULTIPLE REFERENCE POINTS IN RISKY DECISION MAKING
}

\author{
by Gregory Koop
}

Among psychologists and economists, prospect theory continues to be one of the most popular models of decision making. The theory's key property is reference dependence; specifically, how an individual's perception of loss or gain is dependent upon their starting point (i.e. the status quo). Although prospect theory is widely accepted, other authors have sought the inclusion of reference points besides the status quo. Wang and Johnson's (2009) Tri-Reference Point theory (TRP) proposes three reference points: the goal, status quo, and minimum requirement. In two experiments, we present evidence that individuals simultaneously utilize a minimum requirement, status quo, and goal in a risky decision task. Participants most often chose to maximize their chance of reaching reference points even when that decision was riskier, resulted in lower expect value, or resulted in lower expected utility. Furthermore, salience and ambiguity moderated the use of goals and minimum requirements as reference points. 


\title{
THE SIMULTANEOUS USE OF MULTIPLE REFERENCE POINTS IN RISKY DECISION MAKING
}

\author{
A Thesis \\ Submitted to the \\ Faculty of Miami University \\ In partial fulfillment of \\ the requirements for the degree of \\ Master of Arts \\ Department of Psychology \\ by \\ Gregory Koop \\ Miami University \\ Oxford, Ohio \\ 2009 \\ Advisor: Dr. Joseph G. Johnson \\ Reader: Dr. Christopher Wolfe \\ Reader: Dr. Leonard S. Mark
}


Table of Contents

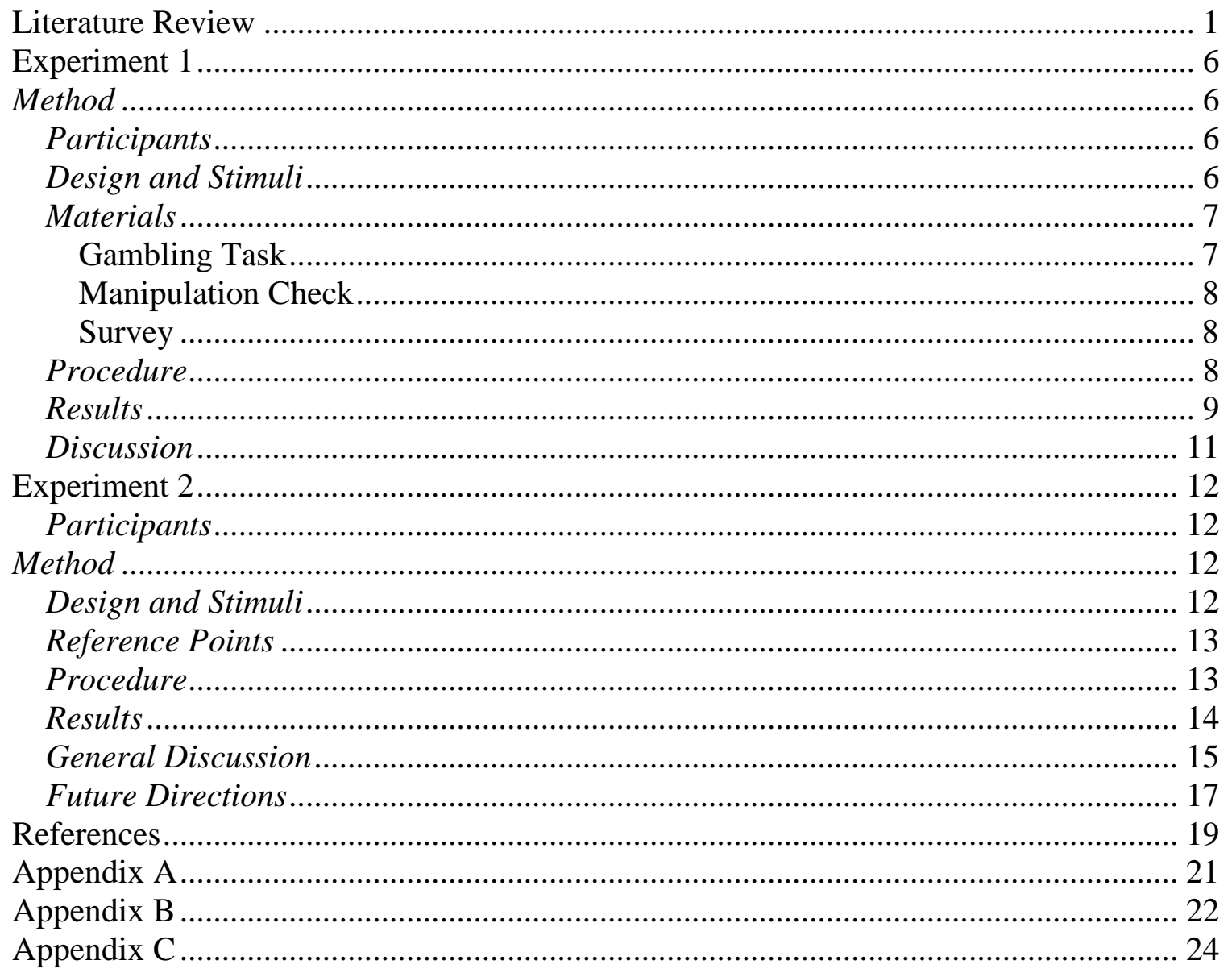




\section{List of Figures}

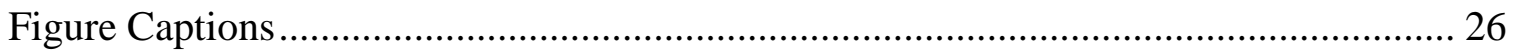

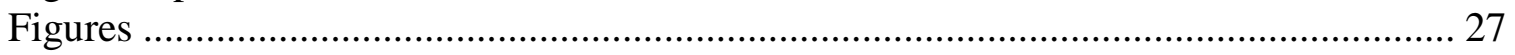

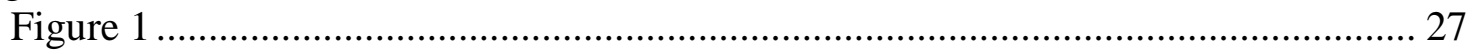

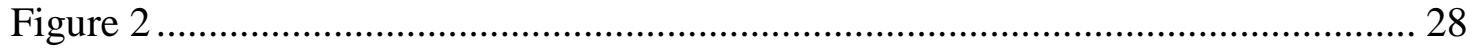

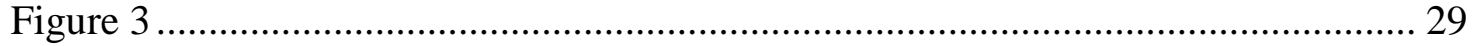

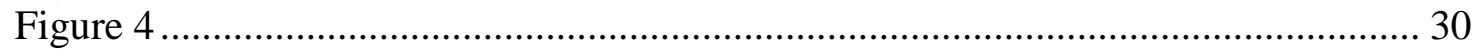

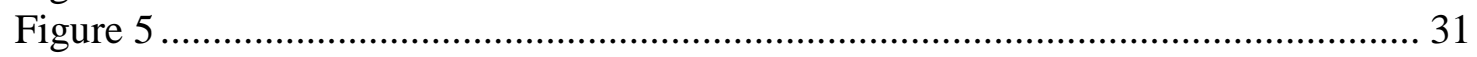




\section{Literature Review}

The study of risky decision making attracts researchers from a variety of fields. Theorists in economics, ecology, and psychology seek to model decision making in situations ranging from financial investments to animal foraging. Some of these theories have been normative - that is, they attempt to model how individuals should respond to a gamble in order to maximize gain. Other theories have been descriptive and model the decisions people actually make. Examples within these categories include expected value, expected utility (Bernoulli, 1738; von Neumann \& Morgenstern, 1947), and most prominently, prospect theory (Kahneman \& Tversky, 1979). Both expected utility and prospect theory included ways to represent subjective facets of human decision making. Specifically, prospect theory links an individual's perceptions of loss or gain to their starting point (i.e., the status quo). The tri-reference point theory (Wang and Johnson, 2008) extends these models and predicts that decision makers will simultaneously utilize reference points in addition to the status quo.

Expected value is one of the oldest and simplest methods for predicting risky choice behavior. To compare choice options, one need only multiply each potential outcome by its probability. Predicting choice is then just a matter of comparing results from this equation. Imagine a person presented with a choice between two gambles, A and B. Gamble A presents a 50-50 chance of winning $\$ 4000$ or $\$ 2000\{4000, .5 ; 2000$, $.5\}$ while gamble $B$ has a $50-50$ chance of winning $\$ 2500$ or $\$ 3500\{2500, .5 ; 3500, .5\}$. Because both gambles have an expected value of $\$ 3000$, a person should have no preference between gambles. However, because of considerations outside of the scope of an expected value calculation, people generally favor one gamble over the other. Although its simplicity is appealing, expected value does not accurately predict behavior.

In an attempt to explain the shortcomings of expected value as a decision making theory, expected utility incorporated the subjective valuation of outcomes (Bernoulli, 1738). This subjective valuation followed the same rules as Weber's law of diminishing marginal sensitivity. In terms of financial gain, moving from $\$ 0$ to $\$ 100$ is subjectively larger than moving from $\$ 400$ to $\$ 500$ even though both represent an objective gain of $\$ 100$ (see Figure 1). Consequently, when given the choice between gamble A $\{2000, .5$; 
$4000, .5\}$ and gamble $\mathrm{B}\{2500, .5 ; 3500, .5\}$, the subjective mean for B may be higher than A even though these gambles have the same expected value. The diminishing marginal utility of outcomes helped to explain why, in some situations, individuals did not make decisions in line with an objective expected value. Von Neumann and Morgenstern (1947) later formalized Bernoulli's concept of expected utility.

In general, individuals were assumed to adhere to a single strategy in forming these subjective valuations. Presented with gambles such as those above, it was assumed that people would be globally risk averse or risk seeking in their gamble preference. However, people often change risk-taking strategies as demonstrated through a wide number of choice problems that systematically violate expected utility principles (Kahneman \& Tversky, 1979).

As demonstrated in the Allais (1953) paradox, people tend to value guaranteed outcomes especially highly even relative to outcomes that are probable, showing a subjective perception of probability (Kahneman \& Tversky, 1979). In response, subjective expected utility theories (Savage, 1954; Edwards, 1962) incorporated subjective probabilities along with the subjective outcomes included in previous expected utility theories. From this line of research, Kahneman and Tversky (1979) proposed arguably the most well known decision making model: prospect theory.

Whereas other subjective expected utility theories allowed for subjective probability (Edwards, 1962; Savage, 1954), prospect theory introduced a number of rules that have guided decision making research since their inception. A key point was that people judged gambles according to relative states rather than objective outcomes (Kahneman \& Tversky, 1979). For example, if a child receiving a \$5 monthly allowance suddenly begins receiving \$3 a month, that child will view the new allowance as a \$2 loss (from the usual \$5) rather than a \$3 dollar gain (from \$0). The child's perception of gain and loss is moderated by the status quo (SQ) of $\$ 5$. Multiple studies have affirmed that people evaluate outcomes relative to their starting point (i.e., the SQ) (Payne, Laughhunn, \& Crum, 1980, 1981; March, 1988; among others). As perceptions of gain and loss change, so do risk-taking strategies. The SQ operates as a reflection point; when gambles are flipped around it (changing gains to losses, and losses to gains) people alter their strategies. In terms of the earlier example, if rather than choosing between two gambles 
representing gains, one had the choice between gamble $\mathrm{A}\{-2000, .5 ;-4000, .5\}$ and gamble $\mathrm{B}\{-2500, .5 ;-3500, .5\}$ risk strategies would likely change. According to prospect theory, people are risk seeking when choosing between gambles resulting in losses and risk averse with equivalent gambles resulting in gains (Kahneman \& Tversky, 1979; Payne, Laughhunn, \& Crum, 1980, 1981). These variable strategies are the product of the diminishing marginal sensitivity mentioned earlier. As one moves closer to the SQ, gains and losses will have larger subjective impacts. In the realm of losses, risk-seeking behavior will see potentially larger subjective gains as one moves towards the SQ whereas in the realm of gains risk-averse behavior will be rewarded to ensure one avoids the larger subjective losses near the SQ.

Prospect theory also showed that the diminishing marginal utility curves introduced by expected utility were not equivalent on either side of the SQ (Kahneman \& Tversky, 1979). Losses have a steeper subjective value curve than gains meaning that losses are weighted more heavily than gains of equal size. Once again, in terms of financial outcomes, a loss of $\$ 100$ is seen as greater than a gain of $\$ 100$. The subjective value of outcomes is dependent upon the outcome's position relative to the SQ.

Although prospect theory posits the status quo as the only important reference point, a number of other studies have shown that reference points besides the status quo can have significant impacts on behavior (e.g. Heath, Larrick, \& Wu, 1999; March \& Shapira, 1992; Lopes \& Oden, 1999). Specifically, research has examined the impact of goals and aspirations as reference points. Both individuals and organizations alter risk taking strategies depending on where they are relative to an aspiration point (March \& Shapira, 1992). Like the status quo in prospect theory, goals can divide outcomes into regions of success and failure, gain and loss (Heath, Larrick, \& Wu, 1999).

Although aspirations are the most prominent example of reference points besides the status quo, survival requirements have also been mentioned as important reference points (March, 1988; Lopes \& Oden, 1999). Survival requirements may refer to the amount of food a foraging animal needs to survive, or the minimum amount of income a worker needs to pay a month's rent. Survival requirements, like goals, exhibit the traits that prospect theory assigned to the status quo. As one moves away from a reference point (whether that be an aspiration, a survival point, or the status quo) the perception of 
value diminishes (Lopes \& Oden, 1999). Additionally, perception of risk will be moderated by their location relative to these reference points (Mao, 1970; March \& Shapira, 1992; Heath, Larrick, \& Wu, 1999). Both aspirations and survival points demonstrate the traits attributed to the status quo; however, when these points are considered together they challenge some of prospect theory's most basic assumptions.

Early theories on multiple reference points suggested people combined these points into a single composite point (e.g. Tryon, 1994; Olson, Roese, \& Zanna, 1996; Ordóñez, 1998) but a more recent study on perceptions of fairness has shown that people can simultaneously consider multiple reference points in their value judgments (Ordóñez, Connolly, \& Coughlin, 2000). The asymmetries in subjective value Kahneman and Tversky attributed to the status quo also appear around these other reference points. Even while people can feel positively relative to one reference point like the status quo they may feel negatively relative to an aspiration (Ordóñez, et al., 2000).

When individuals utilize multiple reference points, unique patterns of risk related behavior emerge. In performance assessment, investment managers have been shown to consider their current level of performance and a target performance rate (Sullivan \& Kida, 1995). Contrary to the tenets of prospect theory, these investment managers were not universally risk averse when their performance was above their status quo. Managers were risk averse when the possibility of falling back to the status quo existed, but when they were guaranteed to stay above the status quo, they tended to be risk seeking in order to reach their targets. Sullivan and Kida hypothesized that when multiple reference points are important for assessment, each of these reference points will concurrently impact behavior.

Additional evidence from animal behavior supports the claim that decision makers use multiple reference points. Animal foraging theory predicts that animals will consider both a starvation threshold and a reproductive energy threshold when making decisions about where to forage for food (Hurly, 2003). Data from hummingbird foraging has shown that these two thresholds moderate risk taking behavior in opposite directions. Around the starvation threshold, animals were risk averse in order to avoid death. Around the reproductive threshold, animals were risk seeking in order to surpass this biologically important reference point (Hurly, 2003). Confirming the work by 
Sullivan and Kida (1995), Hurly's work has shown that prospect theory's assertion of uniform risk seeking for losses and risk aversion for gains is not accurate when one considers multiple reference points. Risk taking behavior can be moderated simultaneously by more than one reference point.

Wang and Johnson (2008) combined these ideas regarding multiple reference points into the tri-reference point (TRP) theory of risky decision making. Although a person can have any number of reference points, Wang and Johnson propose three reference points - the goal $(\mathrm{G})$, the status quo (SQ) and the minimum requirement $(\mathrm{MR})$ - on the basis of 5 criteria. A reference point must be ubiquitous, affect choice preference, reflect specific standards, account for a broad range of biases, and decisions crossing over these points must have greater subjective value than equivalent changes which do not cross a reference point. The three reference points proposed in TRP must meet these criteria in order to evoke reference-point-dependent behavior.

By explicitly considering the effects of three reference points, TRP (Wang \& Johnson, 2008) draws a number of contrasts with prospect theory. Regardless of which side of the status quo a decision maker is on, their decisions will be moderated by the possibility of crossing other reference points. Instead of being universally risk averse for gains or risk seeking for losses as predicted by prospect theory, TRP predicts individuals will show strong risk seeking tendencies when the riskier option allows them the opportunity to move above a reference point. Similarly, people will be risk averse when the safer option guarantees them the opportunity to remain above the reference point. Consider a choice between gamble $\mathrm{A}\{450, .5 ; 550, .5\}$ and gamble $\mathrm{B}\{400, .5 ; 600, .5\}$ where the MR has been set at 0 , the SQ at 300, and the $G$ at 600 . Because gambles A and $\mathrm{B}$ are entirely above the SQ, prospect theory predicts choice of A because it is the less variable option. In contrast, TRP predicts choice of B because it allows the individual to reach the $G$ of 600 .

These divergent predictions result from differences in how diminishing marginal utility is treated in the two theories. Instead of continually decreasing as one moves away from the status quo (as in prospect theory), TRP predicts that marginal differences will increase at the goal and minimum requirement. Although all the reference points will increase marginal differences, the largest change is predicted to be at the MR, followed 
by the G, and finally, the SQ. This assumption is based on evolutionary theory (Wang, 2002) and fits with the idea of "security-first" in business management (e.g., Roy, 1952). Because the minimum requirement is the most important, it should induce the most reference point dependent behavior followed by the goal and the status quo.

Although a wide body of literature offers theoretical support to these TRP predicted behaviors unaccounted for by prospect theory, no one has specifically sought to test the predictions set forth by TRP. Accordingly, the studies presented here induce three reference points within a gambling task and show that these points concurrently moderate individuals' risk strategies. Furthermore, the studies show that individuals will choose gambles predicted by TRP even when in direct competition with more lucrative gambles, prospect theory, or less variable gambles. However, if the reference points lack salience or become more ambiguous (i.e. do not meet the requirements for altering subjective value), the amount of TRP predicted behavior will decrease.

\section{Experiment 1}

\section{Method}

\section{Participants}

College students $(\mathrm{N}=155)$ from introductory psychology classes at a Midwestern university participated in this study. Participants selected the experiment through an online sign-up site that allowed them to choose between many different experiments. For their involvement, participants received course credit and were paid between $\$ 1$ and $\$ 13$ dollars (mode of \$5) based on their decisions, as described below.

\section{Design and Stimuli}

TRP theory functionally divides the outcome space (where $x \in X)$ into failure $(x<$ $\mathrm{MR})$, loss $(\mathrm{MR} \leq x<\mathrm{SQ})$, gain $(\mathrm{SQ}<x<\mathrm{G})$, and success $(\mathrm{G} \leq x)^{1}$. Let $\mathrm{A}=\mathrm{a}_{1} \mathrm{a}_{2}$ represent a single binary gamble A with outcomes $\mathrm{a}_{1}$ and $\mathrm{a}_{2}$. Pairs of binary gambles were created such that, for each pair $\{A, B\}, a_{1}$ and $b_{1}$ were in the same region, whereas $a_{2}$ and $b_{2}$ were in adjacent regions - that is, the second outcomes straddled a reference point (see Figure 2). For example, in Pair 9, both $a_{1}$ and $b_{1}$ represent failure, whereas $a_{2}$ represents a gain

\footnotetext{
${ }^{1}$ We omit here the possible outcome $x=\mathrm{SQ}$, which would simply denote maintenance of the status quo.
} 
and $b_{2}$ represents a success - these outcomes straddle the G. Holding constant the reference point straddled by $\mathrm{a}_{2}$ and $\mathrm{b}_{2}$ and moving $\mathrm{a}_{1}$ and $\mathrm{b}_{1}$ across all functional regions produces four gamble pairs. Repeating this for each of the three reference points produces a total of 4 × $3=12$ gamble pairs seen by each participant. The values of $a_{1}, b_{1}, a_{2}$, and $b_{2}$ were chosen so that A always had a higher expected value, whereas TRP theory predicted preference for the B gamble in each pair due to the better functional outcome. Thus, the dependent variable is the choice frequency for B gambles, which was analyzed separately for each reference point (a within-subjects variable with three levels).

TRP theory also predicts that as reference points become more ambiguous, they will become less salient and are therefore less likely to affect behavior (see Wang \& Johnson, 2008, for a theoretical treatment). To test this prediction, we manipulated the reference point type between-subjects using a fixed condition and a variable condition. In the fixed condition $(\mathrm{N}=72)$, reference points $M R, S Q$, and $G$ were well defined and revealed to participants. In the variable condition $(\mathrm{N}=83)$, SQ was held fixed but MR and $\mathrm{G}$ were expressed in terms of symmetric probability distributions. The mean of each distribution was set equal to the associated value from the fixed condition.

\section{Materials}

We conducted the experiment in a computer lab with participants seated at alternating computers. Participants learned the task through a brief introduction from the experimenter and a computerized presentation that contained detailed instructions for the gambling task as well as information on a bonus drawing that was used to establish the reference points.

\section{Gambling Task}

The gambling task consisted of three identical blocks of the gamble pairings (presented via E-prime software). In order to introduce more variability in the gambles, dollar amounts were represented in lira with an exchange rate of \$1 to 400 lira. Gamble payouts ranged from 20 lira to 5060 lira. Participants were made fully aware that their payout would be determined by the gamble they selected on a randomly chosen trial and that the outcome of that trial would also be randomly determined. Each of the gamble 
pairs had three choice options: an "A" gamble, a "B" gamble, and "Indifferent." For clarity, hereafter let B represent the TRP predicted gamble. However, during the experiment the left/right presentation order was counterbalanced without significant order effects.

\section{Manipulation Check}

In order to ensure participants understood how to earn entries into the bonus drawing (see below), a short quiz followed the instruction slides (see Appendix A, B). The experimenter corrected any mistakes and further explained the system to the participant. Additionally, following each block of gambles, participants took a computerized quiz to verify understanding of the system. We required perfect performance on the quiz to continue to the next block. If a participant failed this quiz three consecutive times, the computer displayed the correct answers and returned the participant to the previous block of gambles. In these instances, data from the initial attempt were discarded and only the new responses were used in analyses.

\section{Survey}

After completing the gambling task, participants immediately filled out a survey asking them about their choice strategies and any goals or requirements they considered. The survey contained both multiple choice and free response questions (see Appendix $\mathrm{C}$ ). These questions were for exploratory purposes and will not be addressed here.

\section{Procedure}

After all participants were seated at their computers, an experimenter introduced the gambling task and notified the participants that their winnings in the task would determine their number of entries into a drawing for a mystery prize worth approximately \$20. During the introduction, the experimenter held a wrapped gift box and placed it in a location where it was visible to all participants. Participants then read through instruction slides at their own pace to learn the final details of the task.

To establish multiple reference points, participants were given various earning benchmarks that determined their entries into the drawing. Participants were told that 
they earned five dollars for showing up at the experiment but could trade those five dollars for the right to play a random gamble from the task. This established a SQ of five dollars (2000 lira). Although the SQ was held constant for all participants, the MR and the $\mathrm{G}$ differed between the two experimental conditions.

In the fixed condition, the MR was set by requiring participants to earn at least 1000 lira to gain any entries in the bonus drawing. After surpassing 1000 lira, the number of entries earned for the bonus drawing was calculated by dividing the amount of lira they earned by 100 (e.g. 1000 lira $=10$ entries). If participants surpassed the $\mathrm{G}$ of 4000 lira, their entries into the bonus drawing would be doubled (e.g. 4000 lira $=80$ entries).

In the variable condition, rather than having a set MR of 1000 and $\mathrm{G}$ of 4000 , participants were told that these points would be randomly selected following a symmetric probability distribution with 1000 lira and 4000 lira as the means. In the variable condition, the MR had a 38\% chance of being between 900 and1100 lira with other possible values ranging from 500 lira to 1500 lira. The $\mathrm{G}$ also had a symmetric distribution with possible values ranging from 3500 lira to 4500 lira (see Figure 3). The number of entries earned in the bonus drawing was actually calculated using the same equation as in the fixed condition.

After completing the instructions, gambling task, and survey, participants went to another room in order to receive their payment. Upon arrival, a single trial from the completed gambling task was randomly chosen. The participant's chosen gamble on that trial was determined from their data and presented to the participant who then had the option of playing that gamble or leaving with the initial five dollars. ${ }^{2}$ If the participant chose to play the gamble, the outcome was randomly selected. The experimenter then paid each participant according to the gamble outcome and calculated the number of entries earned for the bonus drawing, which was performed at a later date. Winners were notified via e-mail and paid $\$ 20$ in cash instead of a mystery prize of that value.

\section{Results}

\footnotetext{
${ }^{2}$ If the participant selected the indifference response on the trial selected for determining payment, then one of the two gambles was randomly selected and played. Participants were told this in the instructions.
} 
The extent to which participants utilized the three reference points (MR, SQ, G) was measured by showing the proportion of times participants chose the TRP gamble when participants were not indifferent. That is, the outcome measure was calculated as $\mathrm{P}(\mathrm{TRP} \mid \sim$ indifferent $)$. These individual proportions were then averaged across subjects. Two participants from the fixed condition were excluded from all analyses due to an unusually high number of indifferent responses (44/48 and 48/48). Chance performance on this task predicts choosing the TRP predicted gamble $50 \%$ of the time.

In the fixed condition, participants showed a significant general tendency to choose the TRP predicted gambles above chance levels $t(70)=7.441, p<.01$. Across all reference points, participants chose the TRP predicted gamble $64.23 \%$ of the time. Over individual reference points - that is, on those gambles where the divergent outcome regions for gambles $\mathrm{A}$ and $\mathrm{B}$ straddled a reference point (e.g., 1, 2, 3, and 4 are gambles around the MR in Figure 2)—participants chose the TRP predicted gamble beyond chance on two of three reference points. Gambles around the MR showed the most TRP predicted behavior with participants choosing the TRP gamble $78.15 \%$ of the time $t(68)=$ $10.984, p<01$. In gambles around the SQ, participants showed a preference for the TRP predicted gamble $t(69)=4.002, p<.01$ and chose it $60.49 \%$ of the time. Unlike the MR and SQ, preference for TRP gambles around the G was not significant $t(69)=-1.802, p=$ .076 as participants only chose the TRP gambles on $44.56 \%$ of gambles.

In the variable reference point condition chance again predicts choosing the TRP predicted B gamble on $50 \%$ of trials. Across all reference points, participants chose the TRP predicted gamble $56.64 \%$ of the time, which was significant beyond chance $t(82)=$ $4.756, p<.01$. Although participants in the variable condition showed a significant tendency towards choosing the TRP predicted gamble overall, these results were not seen over all individual reference points. In gambles around the MR, participants chose the TRP predicted gamble on $68.45 \%$ of trials $t(82)=7.380, p<.01$. Participants did not show a significant tendency to choose the TRP predicted gamble around the SQ, only choosing it $54.01 \%$ of the time $t(82)=1.794, p=.076$. On gambles around the $\mathrm{G}$, participants again significantly differed from chance $t(82)=-5.563$, however they demonstrated a tendency away from the TRP predicted gamble, only choosing it $36.95 \%$ of the time. 
TRP predicts that as reference points become more ambiguous, their impact on behavior will decrease. In both conditions, participants showed an overall preference for the TRP predicted gamble; however, the fixed condition (64.23\%) was significantly higher than preference in the variable condition $(56.64 \%) t(152)=3.266, p<.01$. When these results are further dissected on individual reference points, the data again support the predictions made by TRP. Around the MR, participants in the fixed condition (78.15\%) showed a stronger preference for the TRP predicted gambles than those in the variable condition $(68.45 \%) t(150)=2.691, p<.01$. Around the $\mathrm{G}$, participants in the fixed condition (44.56\%) again showed stronger preference for the TRP gambles than those in the variable condition $(36.95 \%) t(151)=2.019, p=.045$. Differences for gambles around the SQ between the fixed condition (60.49\%) and variable condition $(54.01 \%)$ were not significant $t(151)=1.891, p=.06$. However, this is not surprising, as the SQ did not change between conditions. All participants started with the same amount of money to begin the task thereby creating a fixed SQ, even in the variable condition.

\section{Discussion}

Despite showing an overall preference for the TRP predicted gambles, Experiment 1 did not fully support all of our initial hypotheses. In both conditions, participants failed to show a preference for TRP predicted gambles around the G. A possible explanation is that the $\mathrm{G}$ gambles were confounded by risk-aversion. On three of the four gambles around the G, the TRP gamble was also the riskier (more variable) option. The effect of facing the safer gambles could have excessively decreased preference for the TRP predicted gambles. Also, on gambles around the MR the TRP gambles were safer (less variable) on three of four pairings, which may have inflated preference for the TRP predicted gamble. Without a standardized method for comparing TRP predictions with risk aversion, we were not able to isolate the relative contributions of each strategy. Similarly, it was not possible to systematically compare TRP to prospect theory within Experiment 1.

Despite the various flaws in the initial design, there was an overall trend toward TRP predicted behavior over most reference points in both conditions. However, TRP also predicts an importance hierarchy of reference points (MR $<\mathrm{G}<\mathrm{SQ}$ ), which 
Experiment 1 did not support. It is possible that the reference points were not as salient as they could have been. Specifically, the system for awarding entries into the bonus drawing, although understandable and reiterated in manipulation checks throughout, was not as straightforward as possible. Also, and the "mystery prize" in the bonus drawing may not have interested participants enough to affect behavior.

The limitations of this experiment motivated us to design a second experiment with two key design improvements. First, by controlling when TRP lined up with lowervariance gambles and prospect theory predicted gambles, it became possible to see explicitly whether people utilized decision making strategies based on TRP, risk aversion, or prospect theory. Additionally, by making the reward scheme simpler, it was hypothesized that more reference-point-dependent behavior would emerge. In a second experiment with a revised drawing entry scheme and prize presentation reported below, we show an increase in TRP predicted behavior relative to prospect theory and risk aversion, as well as the predicted hierarchy of reference points within TRP.

\section{Experiment 2}

\section{Participants}

College students $(\mathrm{N}=55)$ from introductory psychology classes at a Midwestern university participated in this task. Selection procedures and compensation were identical to Experiment 1.

\section{Method}

\section{Design and Stimuli}

The division of the outcome space and pairing of gambles were identical to Experiment 1. Once again gamble pairs $\{A, B\}$ held the region of $a_{1}$ and $b_{1}$ in common while $\mathrm{a}_{2}$ and $\mathrm{b}_{2}$ straddled a reference point. This was duplicated over all three reference points creating a set of 12 gambles. Unlike Experiment 1 , the values of $a_{1}, b_{1}, a_{2}$, and $b_{2}$ were chosen specifically to pit TRP, prospect theory, and risk-aversion against one another on a set number of gambles. For each reference point, the TRP predicted gamble (arbitrarily the B gamble) was paired twice with a prospect theory predicted gamble and twice with a risk-aversion predicted gamble (see Figure 4). That is, on two gambles the 
theories made divergent predictions and on two gambles the theories made identical predictions. Again, the dependent variable is choice proportion of B gambles (given a preference besides indifference), which was analyzed separately across each reference point - a within-subjects variable with three levels. Experiment 2 did not contain the variable condition from Experiment 1. In this design, chance behavior predicts $50 \%$ choice of $\mathrm{B}$, prospect theory and risk aversion each predict $50 \%$ choice of $\mathrm{B}$, and TRP predicts $100 \%$ choice of $\mathrm{B}$.

\section{Reference Points}

All reference points were well defined (as in the fixed condition from Experiment 1) and revealed to all participants prior to beginning the gambling task. The values of the MR and $G$ were identical to the fixed condition of Experiment 1. The SQ was changed to 2500 lira in order to center it between the MR and G making the exchange rate $\$ 1=500$ lira.

\section{Procedure}

The overall procedure for Experiment 2 was similar to Experiment 1 but incorporated some minor revisions. Experiment 2 still used a bonus drawing to induce reference points, but the reward and the entry scheme were simplified. Rather than an experimenter presenting a "mystery prize" to participants before the task, participants were informed via instruction slides that the prize for the bonus drawing was a $\$ 20$ gift card to a list of possible stores, from which they could choose. Participants entered their gift card choice immediately prior to beginning the gambling task.

The method for earning entries into the bonus drawing was simplified in order to make the reference points more salient. Participants were told that they had to exceed 1000 lira in order to earn any entries in the drawing. If they surpassed 1000 lira they would earn 5 entries into the bonus drawing and if they surpassed 4000 lira they would earn 10 entries in the drawing. Other than those reference points, the amount of entries in the drawing was not related to amount earned. The manipulation checks, survey, and payment procedure were identical to Experiment 1. 


\section{Results}

Just as in Experiment 1, support for TRP was measured as the proportion of choices for TRP predicted gambles given non-indifference, that is, $\mathrm{P}(\mathrm{TRP} \mid$ indifference). These individual proportions were again averaged across subjects. Chance behavior would predict choosing TRP on 50\% of gamble pairings. Across all gamble pairs, participants showed a strong preference for the TRP gambles and chose the TRP predicted gamble on $71.02 \%$ of gamble pairs $t(50)=11.278, p<.01$.

The general preference for TRP gambles was also present when considering each individual reference point. Around the MR, participants chose the TRP predicted gamble on $81.97 \%$ of pairings $t(50)=13.450, p<.01$. Around the SQ, participants chose the TRP gamble on $64.13 \%$ of pairings $t(50)=6.703, p<.01$. Finally, on gambles around the $\mathrm{G}$, participants chose the TRP gamble on $70.21 \%$ of gamble parings, which was also significant $t(50)=7.669, p<.01$.

Recall in Experiment 1, picking the TRP gamble could have been due to the use of other strategies predicted by risk aversion or prospect theory. The current experiment made it possible to isolate the use of each of these individual strategies by creating series of gamble pairs in which prospect theory, risk aversion, and TRP made divergent predictions. The data revealed behavior strongly in accord with TRP theory and contradictory to those alternative explanations. Participants continued to choose the TRP gambles $63.65 \%$ of the time when pitted against risk-aversion $t(50)=3.719, p<.01$, and $62.25 \%$ of the time when pitted against prospect theory $t(50)=4.929, p<.01^{3}$.

TRP also predicts a hierarchy of reference points. The data show that participants most often picked TRP predicted behavior around the MR (81.97\%), followed by the G $(70.21 \%)$ and the SQ (64.13\%). These differences are in line with our predicted importance hierarchy of $M R>G>S Q$. Planned comparisons confirmed that there was significantly greater TRP behavior elicited around the MR than the $\mathrm{G}(\mathrm{MR}>\mathrm{G}) t(50)=$ 4.496, $p<.01$ as well as more TRP behavior elicited around the G than the SQ $(\mathrm{G}>\mathrm{SQ})$ $t(50)=2.078, p=.022$.

\footnotetext{
${ }^{3}$ Out of the 12 unique gamble pairs, TRP made divergent predictions with PT on 6 gambles and with risk aversion on 6 gambles. Only the 6 gambles where theories made different predictions were included in this analysis.
} 


\section{General Discussion}

Wang and Johnson's (2008) Tri-Reference Point theory of risky decision making makes a number of predictions that have been largely untested. Primarily, TRP predicts that individuals can simultaneously utilize reference points other than the status quo when making risky decisions. In Experiment 1, participants demonstrated awareness of multiple reference points by choosing risk-taking strategies predicted by TRP. Regardless of being in the realm of gains or losses, participants were generally riskseeking when they had the possibility of surpassing a reference point and risk-averse when in danger of falling below one. These behaviors demonstrate an increase in descriptive power in TRP over prospect theory; however, Experiment 1 did not allow for a direct comparison between the two theories.

Experiment 2 directly examined many of the questions implied by the results of Experiment 1. Experiment 2 systematically compared TRP predictions to those made by prospect theory and risk aversion. Experiment 2 replicated the initial findings from Experiment 1 that across all gamble types (e.g. choice between a loss-loss gamble and a loss-gain gamble), individuals made choices in accordance with three reference points: the G, SQ, and MR. With a simplified structure and more salient goals, Experiment 2 showed individuals strove to cross or stay above each of the three reference points. In short, these data show that individuals constructed a more nuanced idea of success and did not simply divide the outcome space along the SQ. On gambles where prospect theory and TRP made divergent predictions, the data from Experiment 2 confirmed that individuals chose gambles in line with TRP rather than prospect theory. Similarly, individuals also preferred TRP gambles to a general risk-averse strategy.

While showing a strong preference for TRP gambles across all reference points, Experiment 2 also demonstrated the predicted importance hierarchy of $M R>G>S Q$; that is, the MR is the most important in affecting behavior, followed by the G. This hierarchy fits with evolutionary theory and the business mantra of "safety first" (Wang, 2002). The SQ, being least important in the hierarchy, suggests that although the general principles of prospect theory are sound, a strict adherence to status-quo-dependent decreasing marginal utility may be oversimplified as an explanation of how people perceive and judge success. 
The idea that an individual may be confronted with multiple reference points when making a decision is not a new one. Although decision literature has acknowledged multiple reference points, much of this research assumed these reference points would be combined into a single, composite reference point that would behave similarly to the status quo (Tryon, 1994; Olson, Roese, \& Zanna, 1996; Ordóñez, 1998). However, results from experiments 1 and 2 distinctly show that individuals can keep these reference points separate and will utilize each of them to inform risk-taking strategies.

Traditionally, similar findings have come from outside of the decision literature (Heath, Larrick, \& Wu, 1999). Literature concerning aspirations and survival requirements has previously utilized multiple separate reference points (e.g., March \& Shapira, 1992; Heath, Larrick, \& Wu, 1999; March, 1988; Lopes \& Oden, 1999). At the very least, the aspiration literature has shown that individuals can judge success and failure differently relative to two simultaneously held reference points (Ordóñez, 2008). In decision making research, the possibility of multiple reference points has been largely neglected (although Lopes (1987), Higgins (1997) and others have incorporated similar concepts as motivational influences). Our findings, while novel within the area, support and extend a substantial body of findings from related fields.

Specifically, these results offer strong support for Wang and Johnson's (2008) trireference point theory and the critiques of prospect theory contained therein. Rather than a complete overhaul of prospect theory (1972), our findings support an increasingly nuanced theory espousing many of the same principles. For example, the concept of diminishing marginal utility continues to describe the relationship between subjective value and objective gain. However, instead of a lone marginal increase at the status quo, increases also occur around the MR and the G.

A possible critique of our findings is that we have simply induced artificial reference points without any real world counterpart. Fortunately, several real world examples of imposed reference points support our methodology. Sullivan and Kida's (1995) study with investment bankers dealing with company mandated quotas produced similar findings. Their results showed variable risk-taking strategies within the realm of gains causing them to conclude, "risky decision making may be more complex than 
models such as Prospect Theory would suggest in certain decision contexts" (Sullivan \& Kida, 1995, p. 82). Their findings within the decision context of investment banking demonstrate that imposed multiple reference points are at least plausible in real world settings.

More recently, Wang and Johnson (2008) utilized the standard corporate salary structure in Shanghai, China to support TRP. In Shanghai, it is common for people to be offered jobs on a fixed salary, a flexible (high-variance) salary, or on an intermediate (low-variance) salary. The authors asked college seniors how much they would need to earn in order to survive in Shanghai (MR) as well as what they hoped to earn in their first job $(\mathrm{G})$. These values were consistent across different experimental groups. In accordance with our findings, Wang and Johnson showed that college students chose payment plans depending on their ability to surpass these elicited reference points.

Even more aptly, our results show that behavioral data from hummingbird foraging represents constructs similar to those found in human beings (Hurly, 2003). Hurly showed that hummingbirds became more risk averse when approaching a survival requirement (our MR) and risk seeking when approaching a reproductive threshold (our G). Although there has not be research with plausible human situations directly assessing the TRP model, other research does support the possibility of TRP to apply to everyday settings.

\section{Future Directions}

Despite this support, more research should be done to bring TRP into reasonably lifelike paradigms. Future research should include everyday scenarios that will portray situations that could ostensibly be a common occurrence in the lives of participants. Specifically, these paradigms could include work with setting budgets or with goals for student grades during the semester. Using similar paradigms, it will not be possible to completely avoid imposing reference points but working under mandated quotas is commonplace. However, these reference points should be less artificial than they were in the present experiment.

In life, when these reference points or quotas are imposed individuals will often have autonomy in deciding either where to set these reference points or in what manner 
they will strive to achieve them. Research into aspirations has shown performance improves when participants are asked to set their own specific, challenging goal (Heath, Larrick, \& Wu, 1999). Giving participants the opportunity to set their own goals will not only increase interest in the experiment thereby providing better data, but could also show an increase in TRP predicted behavior. By providing more autonomy in realistic scenarios, it should be possible to increase the both the effect size and descriptive capabilities of TRP. 


\section{References}

Allais, M. (1953). Le comportement de l'homme rationnel devant le risque: critique des postulats et axiomes de l'école Américaine, Econometrica, 21, 503-546.

Bernoulli, D. (1738/1954). Exposition of a new theory on the measurement of risk. Econometrica, 22, 23-36.

Edwards, W. (1962). Subjective probabilities inferred from decisions. Psychological Review, 69, 109-135.

Heath, C., Larrick, R.P., \& Wu, G. (1999). Goals as reference points. Cognitive Psychology, 38, 79-109.

Higgins, E.T. (1997). Beyond pleasure and pain. American Psychologist, 52, 1280-1300.

Hurly, A.T. (2003). The twin threshold model: risk-intermediate foraging by rufous hummingbirds, Selasphorus rufus, Animal Behaviour, 66, 751-761.

Kahneman, D., \& Tversky, A. (1979). Prospect theory. Econometrica, 47, 263-292.

Lopes, L.L. (1987). Between hope and fear: The Psychology of risk. Advances in Experimental Social Psychology, 20, 255-295.

Lopes, L. L., Oden, G. C. (1999). The role of aspiration level in risky choice: a comparison of cumulative prospect theory and SP/A theory. Journal of Mathematical Psychology, 43, 286-313.

Mao, J.C.T. (1970). Survey of capital budgeting: theory and practice. Journal of Finance, 25, 349-360.

March, J.G. (1988). Variable risk preferences and adaptive aspirations. Journal of Economic Behavior and Organization, 9, 5-24.

March, J.G., \& Shapira, Z. (1992). Variable risk preferences and the focus of attention. Psychological Review, 99, 172-183.

Olson, J.M., Roese, J.J. \& Zanna, M.P. (1996). Expectancies. In Higgins, E.T. \& Kruglanski, A. (eds.), Social Psychology: Handbook of Basic Principles (pp. 211-238), New York: Guilford Press.

Ordóñez, L.D. (1998). The effect of correlation between price and quality on consumer choice. Organizational Behavior and Human Decision Processes, 75, 258-273. 
Ordóñez, L.D., Connolly, T., \& Coughlin, R. (2000). Multiple Reference points in satisfaction and fairness assessment. Journal of Behavioral Decision Making, 13, 329-344.

Payne, J.W., Laughhunn, D.J., \& Crum, R. (1980). Translation of gambles and aspiration-level effects in risky choice behavior. Management Science, 26, 10391060.

Payne, J.W., Laughhunn, D.J., \& Crum, R. (1981). Further tests of aspiration level effects in risky choice behavior. Management Science, 27, 953-958.

Roy, A.D. (1952). Safety first and the holding of assets. Econometrica, 20, 431-449.

Savage, L. J. (1954). The foundations of statistics. New York: Wiley.

Sullivan, K., \& Kida, T. (1995). The effect of multiple reference points and prior gains and losses on managers' risky decision making. Organizational Behavior and Human Decision Processes, 64, 76-83.

Tryon, W.W. (1994). Expectation. In Ramachandran, V.S. (ed.), Encyclopedia of Human Behavior, San Diego, CA: Academic Press.

von Neumann, J., \& Morgenstern, O. (1947). Theory of games and economic behavior. Princeton, NJ: Princeton University Press.

Wang, X.T. (2002). Risk as reproductive variance. Evolution and Human Behavior, 23, $35-57$.

Wang, X.T., \& Johnson, J.G. (2008). Tri-reference point theory of risky choice. Manuscript under review. 


\section{Appendix A}

\section{Manipulation Check for Experiment 1a \& Experiment 2}

How many lira must you win today in order to receive any entries into the bonus drawing?

How many lira must you win today in order for your number of entries to be doubled?

If you win 800 lira today, how many entries would you earn in the bonus drawing?

If you win 2,800 lira today, how many entries would you earn in the bonus drawing?

If you win 4,800 lira today, how many entries would you earn in the bonus drawing?

Sub \# 


\author{
Appendix B \\ Manipulation Check for Experiment 1b
}

\title{
Please enter your answers to the following questions in the spaces provided.
}

What is the range of possible values for the lower goal?

to

What is the range of possible values for the upper goal?

to

The most likely value for the lower goal is...

The most likely value for the upper goal is...

If you win 800 lira today, how many entries would you earn in the bonus drawing if...

... the lower goal is randomly selected to be 700 ?

... the lower goal is randomly selected to be 900 ?

If you win 4,200 lira today, how many entries would you earn in the bonus drawing if...

... the upper goal is randomly selected to be 4,100 ?

... the upper goal is randomly selected to be 4,300 ?

Sub \# 


\section{Page 2}

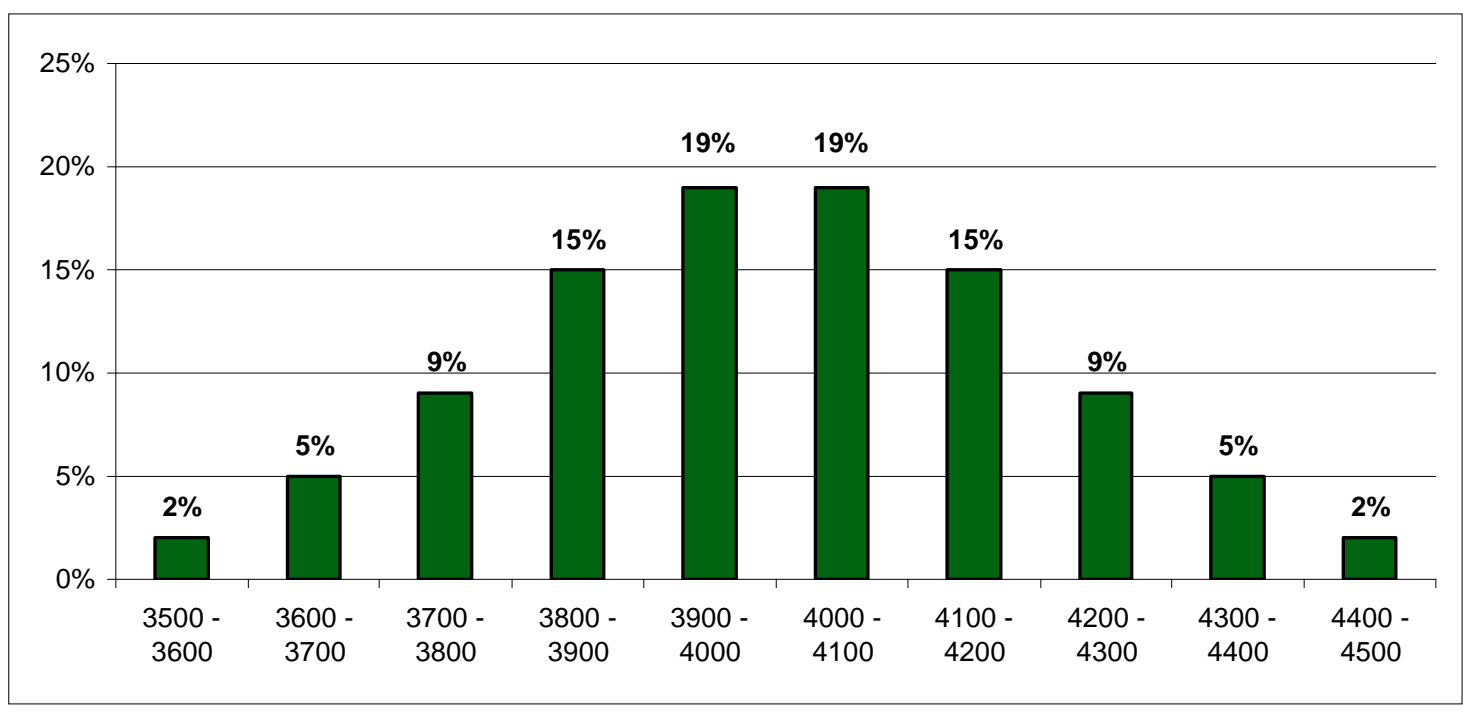

Possible Lira Amounts Needed to Earn Entries in Drawing

(lower goal)

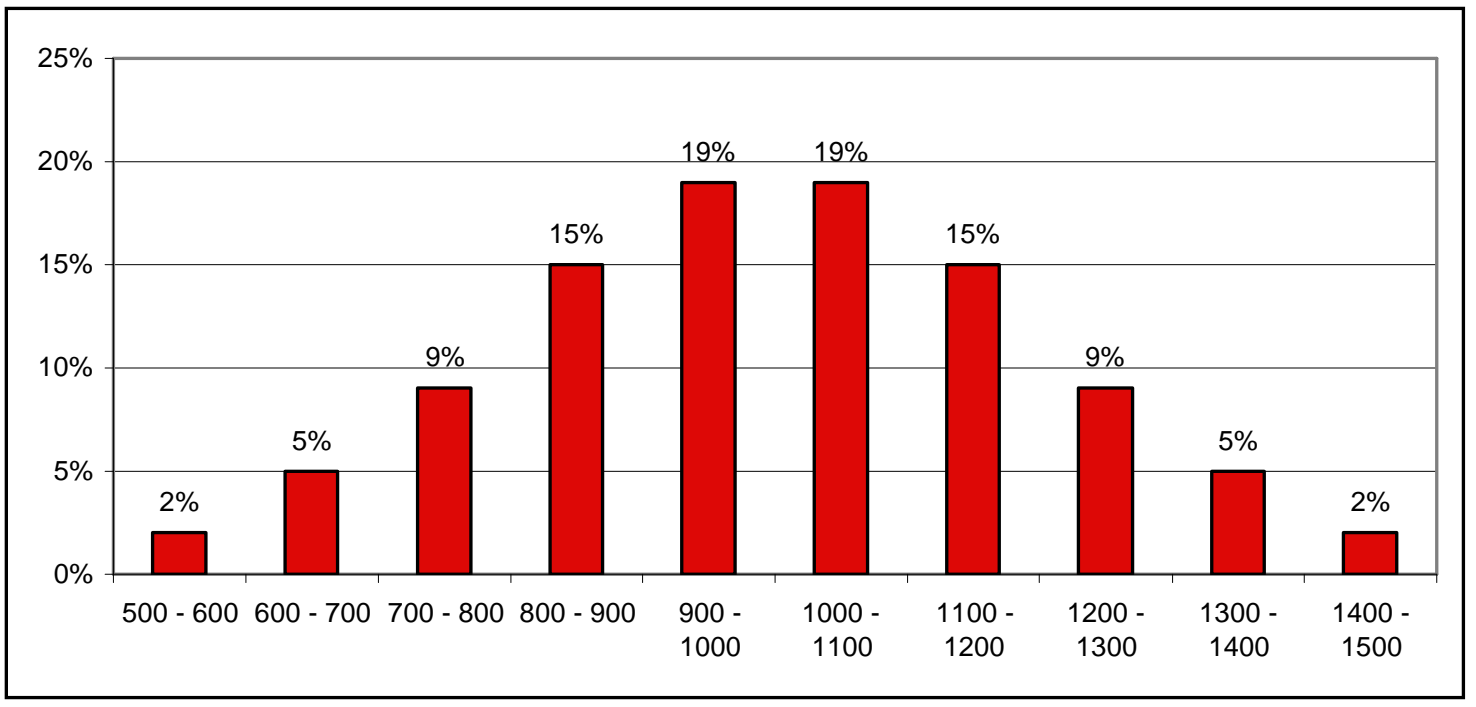

Possible Lira Amounts Needed to Double Entries in Drawing (upper goal) 


\section{Appendix C \\ End Survey}

\section{Did you use any specific strategies when choosing among gambles?}

Please indicate in the box below any methods you may have used in making your choices, to the extent that you in fact used any sort of strategies and can verbalize them. There are no right or wrong answers, we are just curious about how you went about making your decisions.

\section{Did you use the following strategies or rules?}

Please select from the following rules the one that best describes how you made the majority of your choices. There are no right or wrong answers; again, we are just curious how you might have made the decisions you did.

1. I chose primarily to obtain the highest possible outcome

2. I chose primarily to avoid the lowest possible outcome

3. I chose primarily to obtain the best average outcome

4. I did not consistently use any of the above rules/strategies

\section{Did you have any goals or requirements?}

Please indicate in the box below any goals or requirements that you may have had in mind when making your choices, to the extent that you in fact had any sort of goals and can verbalize them. There are no right or wrong answers, we are just curious about how you went about making your decisions.

\section{Which of the following statements describe how you made your decisions?} Please check all that apply

1. I wanted to make as much money today as possible

2. I wanted to make at least the initial amount (2500 lira) ${ }^{4}$

3. I wanted to avoid losing as much as possible

4. I wanted to at least gain some entries into the bonus drawing

\footnotetext{
${ }^{4}$ The value of 2500 lira was only used in Experiment 2. During Experiment 1 participants saw the corresponding SQ of 2000 lira.
} 
5. I wanted to gain as many entries as possible in the bonus drawing

6. I wanted to double my entries in the bonus drawing

7. I just wanted to get this task over with

\section{Finally, on the following screen are several statements. Please rate each of the} statements based on how well they describe how you made the decisions that you did.

1. I was very concerned with gaining entry into the bonus drawing

2. I was very concerned with earning as much money today as possible

3. I was very concerned with gaining a high number of entries into the bonus drawing

4. I was very concerned with earning at least 2500 lira today (the initial amount mentioned)

5. I was very concerned with not earning a small amount (i.e., avoiding gambles with very low outcomes)

6. I was very concerned with doubling my number of entries into the bonus drawing 


\section{Figure Captions}

Figure 1. The concept of diminishing marginal sensitivity in terms of financial gain. An objective financial gain from $\$ 0$ to $\$ 100$ is subjectively greater than a financial gain from $\$ 400$ to $\$ 500$.

Figure 2. The distribution of gamble outcomes across reference points. Each gamble in gamble pair $\{A, B\}$ has two outcomes $\left\{a_{1}, a_{2}\right\}$ and $\left\{b_{1}, b_{2}\right\}$. These outcomes are distributed so that for $\{A, B\}$, outcomes $a_{1}$ and $b_{1}$ are in the same region while $a_{2}$ and $b_{2}$ straddle a reference point. In gambles $1-4$, outcomes $\mathrm{a}_{2}$ and $\mathrm{b}_{2}$ straddle the MR. In 5-8, these outcomes straddle the SQ, and in 9-12 they straddle the G.

Figure 3. The distributions for the $M R$ and the $G$ in the variable condition. Participants were told the probability that MR and G would assume any of these values.

Figure 4. The division of predictions made by TRP, prospect theory, and risk-aversion. Here, four gambles, each with different prediction combinations, are repeated across each of the three reference points. "Gamble Number" refers to the gamble represented in Figure 2. In this subset of four gambles, TRP predicts $100 \%$ choice of B, prospect theory and risk aversion predict $50 \%$ choice of $\mathrm{B}$, and chance also predicts $50 \%$ choice of $\mathrm{B}$.

Figure 5. Mean conditional proportion of choosing the TRP predicted gamble across each of the reference points. Table shows the predictions made by each theory on each set of gambles. All choice proportions were significantly different from chance. 
Figures

Figure 1

\section{Subjective}

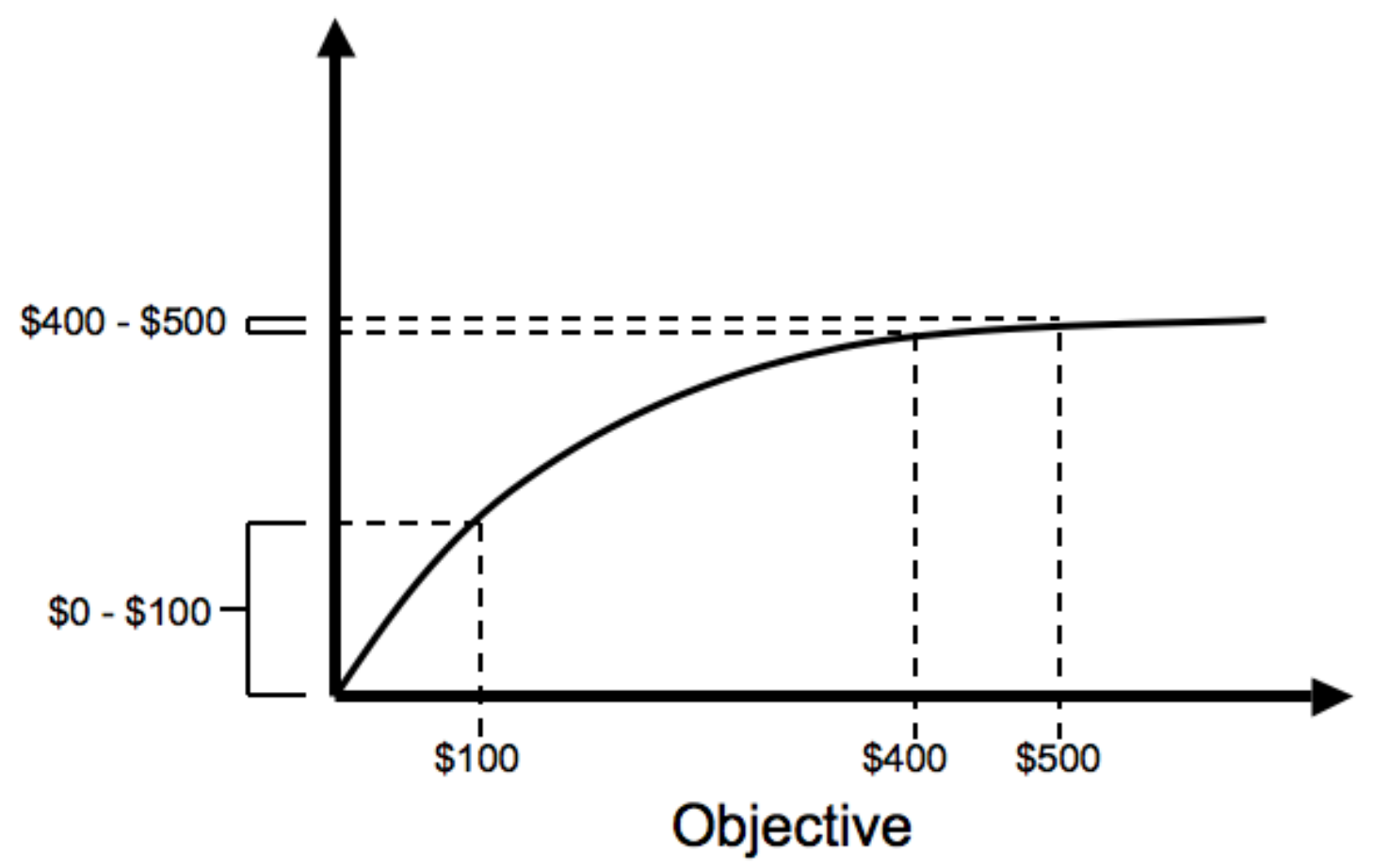


Figure 2

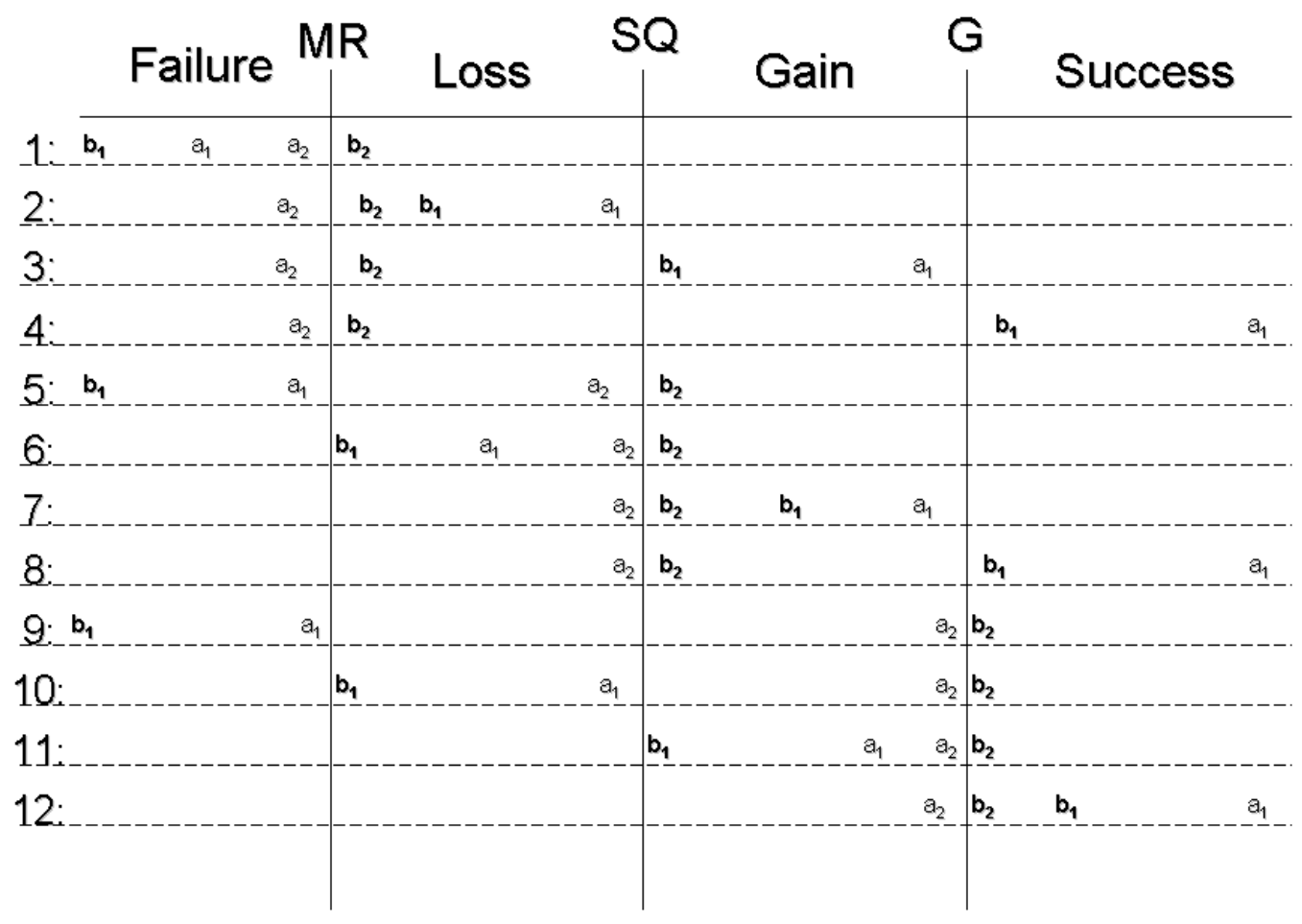




\section{Figure 3}
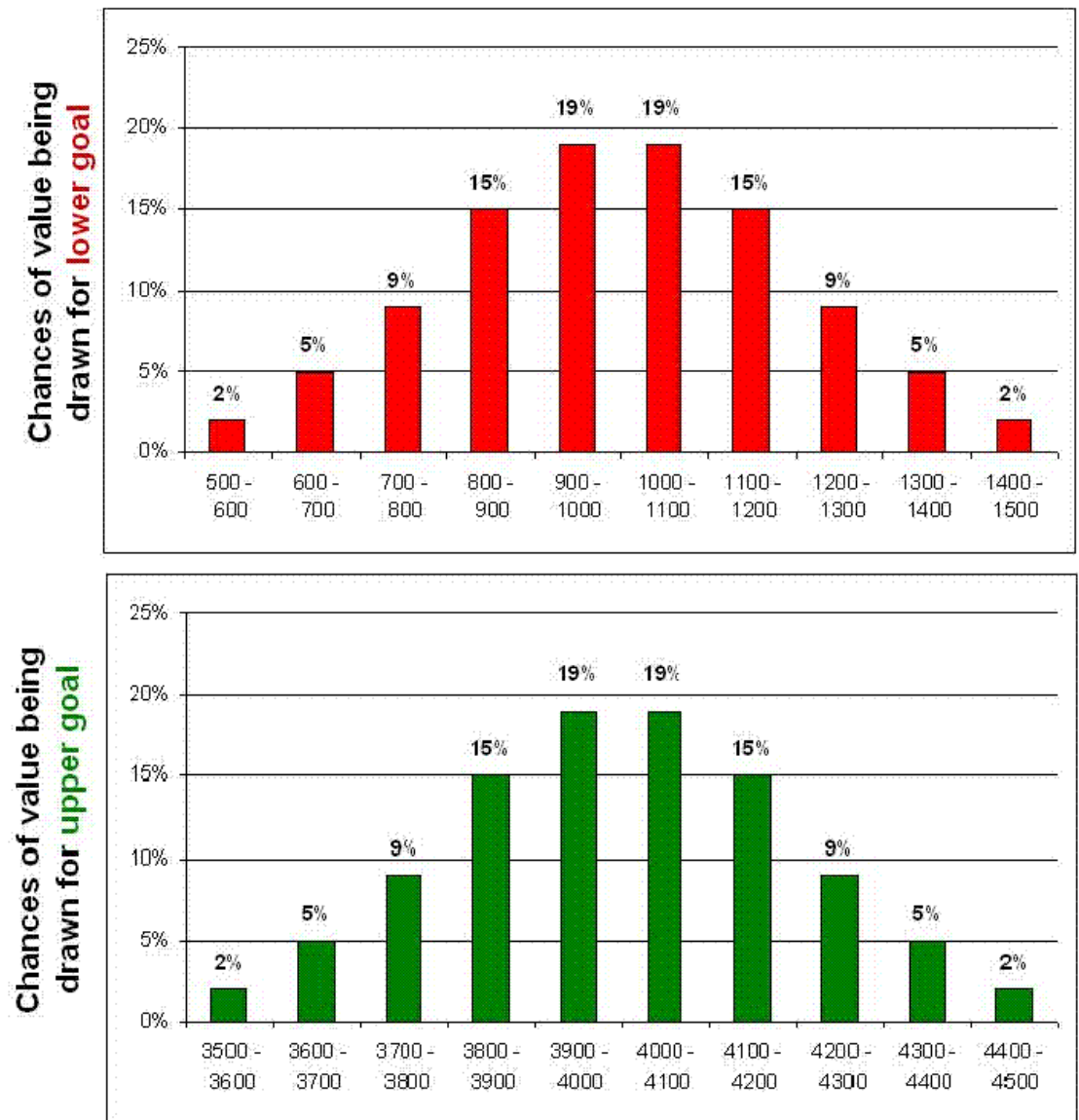
Figure 4

\begin{tabular}{|l|l|l|}
\hline Gamble A & Gamble B & Gamble Number (Fig. 2) \\
\hline Prospect Theory, Low-Variance & TRP & $1,6,11$ \\
\hline Prospect Theory & TRP, Low-Variance & $2,7,12$ \\
\hline Low-Variance & TRP, Prospect Theory & $4,5,10$ \\
\hline & TRP, Prospect Theory, Low-Variance & $3,8,9$ \\
\hline
\end{tabular}


Figure 5

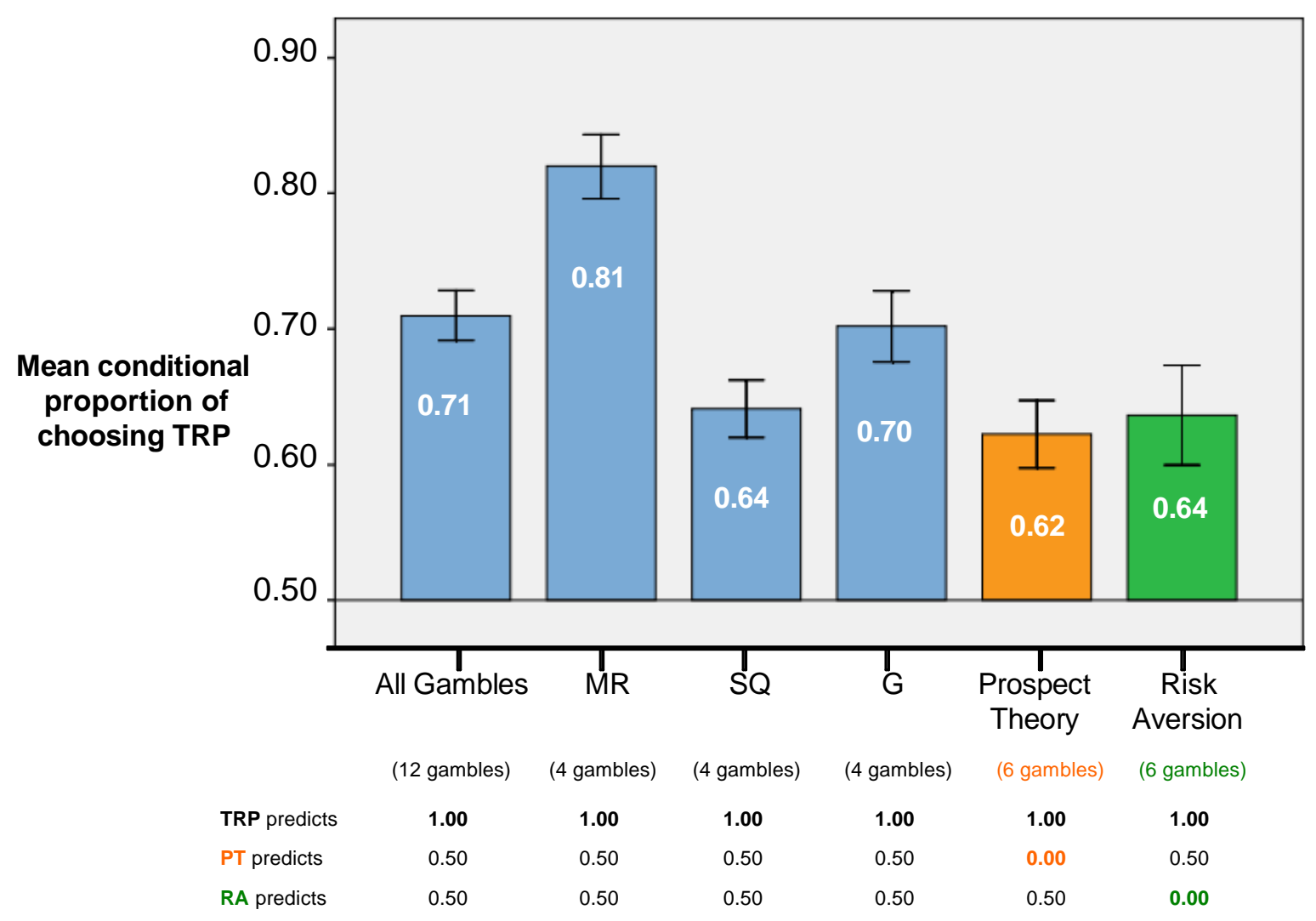

